\title{
Aspectos sociocognitivos asociados al uso de videojuegos colaborativos y violentos*
}

\author{
Rolando Pérez-Sánchez ${ }^{1}$ \\ Gloriana Giusti-Mora² \\ Karina Soto-Chavarría ${ }^{3}$
}

Recibido: 01/02/2019

Aprobado por pares: 11/06/2019
Enviado a pares: 01/02/2019

Aceptado: 17/06/2019

DOI: 10.5294/pacla.2020.23.2.4

\section{Para citar este artículo / to reference this article / para citar este artigo}

Pérez-Sánchez, R., Giusti-Mora, G. y Soto-Chavarría, K. (2020). Aspectos sociocognitivos asociados al uso de videojuegos colaborativos y violentos. Palabra Clave, 23(2), e2324. https://doi.org/10.5294/pacla.2020.23.2.4

\section{Resumen}

El estudio se dirige a investigar las diferencias en el uso de un videojuego colaborativo y un videojuego violento a nivel sociocognitivo. Específicamente interesa indagar las diferencias en la percepción del estado de ánimo, el transporte emocional y el comportamiento altruista, así como los factores motivacionales relacionados con el uso de los videojuegos que permiten comprender las variaciones en estas tres dimensiones. Se llevó a cabo un experimento con 50 estudiantes universitarios, el $48 \%$ mujeres y una edad promedio de 20,6 (DT = 1,83). Como procedimiento de análisis se recurrió a pruebas ty a análisis de regresión lineal jerárquica, método paso a paso (stepwise). Los resultados muestran evidencias de que jugar uno $\mathrm{u}$ otro juego se asocia a diferentes respuestas sociocognitivas, sobre todo en el

\footnotetext{
* Artículo financiado por la Vicerrectoría de Investigación de la Universidad de Costa Rica. Número: 723-B4-312

$1 \bowtie$ https://orcid.org/0000-0001-6321-2543. Universidad de Costa Rica, Costa Rica. rolaando.perez@ucr.ac.cr https://orcid.org/0000-0002-5323-077X. Universidad de Costa Rica, Costa Rica.

https://orcid.org/0000-0002-5251-9766. Universidad de Costa Rica, Costa Rica.
} 
plano emocional y motivacional, hallazgos relevantes para la comprensión de los usos cotidianos de estas tecnologías. Se encontró que el videojuego violento favorece estados de ánimo más positivos, mientras que el videojuego colaborativo evoca mayor transporte emocional. Asimismo, la necesidad psicológica de filiación se asocia con el acto de videojugar en contextos de juego compartido, independiente del tipo de juego.

\section{Palabras clave (Fuente: tesauro de la Unesco)}

Videojuego colaborativo; videojuego violento; cognición social; motivación; emoción. 


\section{Socio-Cognitive Aspects Associated with Collaborative and Violent Video Games*}

\section{Abstract}

The study aims at researching into the socio-cognitive differences between playing a collaborative video game and a violent video game. Specifically, it is interesting to identify the dissimilarities in the perception of mood, emotional immersion, and altruistic behavior, as well as the motivational factors related to playing video games that allow understanding the variations in these three dimensions. An experiment was carried out with 50 university students, $48 \%$ women and an average age of $20.6(\mathrm{SD}=1.83)$. As an analysis procedure, $t$-tests and hierarchical linear regression were used (stepwise method). Results reveal that playing one game or the other is associated with diverse socio-cognitive responses, especially on the emotional and motivational levels. These findings are relevant to comprehending the daily uses of these technologies. The violent video game was found to favor more positive moods, while the collaborative one induces greater emotional immersion. The psychological need for connection is also associated with the act of playing video games in shared contexts, regardless of the type.

\section{Keywords (Source: Unesco Thesaurus)}

Collaborative video games; violent video game; social cognition; motivation; emotion.

* Article funded by the Office of the Vice President for Research, Universidad de Costa Rica. Number: 723-B4-312 


\section{Aspectos sociocognitivos associados com o uso de videogames colaborativos e violentos*}

\section{Resumo}

O objetivo deste estudo é pesquisar as diferenças no uso de um videogame colaborativo e um videogame violento no âmbito da cognição social. Em específico, interessa questionar as diferenças na percepção do estado de humor, no transporte emocional e no comportamento altruísta, bem como os fatores motivacionais relacionados com o uso de videogames que permitem compreender as variações nessas três dimensões. Realizou-se uma experiência com 50 estudantes universitários - $48 \%$ mulheres e idade média de 20,6 anos $(D P=1,83)$. Como procedimento de análise, recorreu-se a testes t e à análise de regressão linear hierárquica, método passo a passo (stepwise). Os resultados mostram evidências de que jogar um ou outro jogo está associado com diferentes respostas cognitivas, sobretudo no plano emocional e motivacional, achados relevantes para compreender os usos cotidianos dessas tecnologias. Verificou-se que o videogame violento favorece estados de humor mais positivos, enquanto o colaborativo evoca maior transporte emocional. Além disso, a necessidade psicológica de afiliação se relaciona com o ato de jogar em contextos de jogos em rede, independentemente do tipo de jogo.

\section{Palavras-chave (Fonte: tesauro da Unesco)}

Videogame colaborativo; Videogame violento; cognição social; motivação; emoção. 


\section{Introducción}

Este estudio se dirige a estudiar las diferencias en el uso de un videojuego colaborativo con respecto a un juego violento a nivel sociocognitivo. Específicamente, interesa estudiar las diferencias en la percepción del estado de ánimo, el transporte emocional, el comportamiento prosocial, así como los factores motivacionales relacionados con el uso de los videojuegos que permiten comprender las variaciones en estas tres dimensiones.

La selección de esta temática se basa en el modelo integrado de evaluación y selección de los contenidos mediáticos (Lewis, Tamborini \& Weber, 2014) que supone que las personas usan los medios tanto para la consecución de disfrute como para la apreciación de los contenidos. Esto sugiere la integración del modelo teórico hedonista y el eudaimónico.

El modelo hedonista supone que las personas se orientan a usar los medios con el objetivo de regular sus estados de ánimo, en busca de aquellos contenidos que le permitan orientarse hacia afectos positivos (Bryant, Eweldosen \& Cantor, 2003). Esta perspectiva se enmarca dentro de la teoría del manejo de las emociones de Zillmann (1988), una de las perspectivas que más ha influenciado el estudio del uso mediático entendido como entretenimiento.

El modelo eudaimónico proviene de la ética aristotélica que señala que la consecución de la felicidad puede ser alcanzada a través de la virtud (Eden, Grizzard \& Lewis, 2013). A efectos de la psicología de medios, se trata de los usos tecnológicos orientados según criterios morales asociados a empatía, prosocialidad, justicia, crítica social (Oliver \& Bartsch, 2010).

Por esta razón, es de interés del estudio conocer las variaciones a nivel sociocognitivo implicadas en el uso de tipos de videojuegos tanto violento como colaborativo. Se parte, entonces, del supuesto de que un videojuego de tipo violento evoca un tipo de uso fundamentalmente hedonista, mientras que un videojuego de tipo colaborativo se va a vincular a un uso fundamentalmente eudaimónico, por cuanto el videojuego típicamente violento se dirige al daño físico entre los personajes como manera de supervivencia 
o triunfo en el juego, en tanto que los videojuegos colaborativos no violentos se orientan a la resolución conjunta de tareas o problemas sin que medie la agresión o daño hacia otros personajes (Greitemeyer \& Osswald, 2009).

Según muestra el metaanálisis realizado por Anderson et al. (2010), el videojuego con contenido violento se asocia de manera positiva con la conducta agresiva y con la cognición y el afecto agresivo, resultados que han sido respaldados por estudios experimentales, transversales y longitudinales. Esta exposición se relacionó con la desensibilización y la falta de empatía y de conducta prosocial, lo cual confirma que sus efectos a largo plazo suelen ser nocivos para ambos sexos, pues no hubo diferencias en cuanto a la susceptibilidad.

Por su parte, Greitemeyer, Agthe, Gschwendther \& Turner (2012), encontraron que la exposición a videojuegos con contenido violento aumentaba la conducta agresiva, mientras que los videojuegos prosociales generaban conductas menos agresivas en los participantes, incluso este resultado se mantuvo para aquellos que jugaron videojuegos neutrales, es decir, aquellos en los que no está presente contenido prosocial o agresivo (Hollingdale \& Greitemeyer, 2014). Este efecto permaneció estadísticamente significativo aun controlando el estado anímico de los participantes y la excitación o arousal percibido. Asimismo, en su segundo experimento, encontraron que los videojuegos prosociales no solo decrecen la conducta agresiva de manera directa, sino también indirectamente, tanto en la cognición como en el afecto, variables que en lo que respecta a la agresión también decrecen.

En relación con los resultados encontrados por los autores mencionados, Greitemeyer \& Osswald (2009) realizaron dos estudios en los que encontraron evidencia que respalda la hipótesis de que jugar videojuegos colaborativos en comparación con neutrales disminuye las respuestas agresivas. Lo anterior se da porque jugar el videojuego colaborativo reduce la expectativa de que otras personas respondan con pensamientos, sentimientos y conductas agresivas, además de disminuir la accesibilidad de pensamientos antisociales. 
Con respecto a los efectos de los videojuegos con contenido colaborativo propiamente, se ha encontrado que el uso de videojuegos colaborativos se asocia a mayores puntajes de empatía y a una disminución del placer que pueda generar la desgracia o el sufrimiento de otra persona (Greitemeyer, Osswald \& Brauer, 2010). De igual forma, este tipo de contenido reduce la hostilidad y los sentimientos negativos, y simultáneamente aumenta el afecto positivo en comparación con videojuegos violentos e, incluso neutrales (Saleem, Anderson \& Gentile, 2012).

En este estudio, no se consideran los videojuegos prosociales violentos, ya que, a diferencia de los exclusivamente violentos, el fin último no es causar daño a otra persona, sino recurrir a la violencia como un medio altruista superior (salvar a una persona, ciudad o planeta, por ejemplo) (Jerabeck \& Ferguson, 2013).

Los anteriores estudios permiten aportar evidencias para sostener que los tipos de videojuegos, o violentos o prosociales, refieren a diferentes tipos de procesamiento sociocognitivo y emocional. No obstante, esta investigación no parte de un modelo determinista que supone un efecto directo de los videojuegos violentos sobre las conductas agresivas. $\mathrm{Al}$ respecto, se presenta en la literatura un debate relevante. Así Fergusson \& Beresin (2017) o Drummond, Sauer \& Garea (2018) hacen una revisión de varios metaanálisis señalando que la asociación entre videojuego y conducta violenta presenta un poder estadístico reducido. No obstante, no se descarta la presencia de una asociación, solo que mediada más bien por factores contextuales, familiares o de personalidad, por ejemplo. Al respecto, en este trabajo, se parte de la propuesta de Fergusson \& Beresin (2017), quienes sostienen que el estudio psicosocial de los videojuegos debe realizarse desde un modelo motivacional, que permite considerar a las personas jugadoras en tanto agentes que orientan su uso según diferentes motivos.

A partir de lo señalado, es que se considera relevante investigar el estado de ánimo, el transporte emocional y el altruismo, dimensiones destacadas para indagar las diferencias entre uno y otro tipo de videojuego. Precisamente, al considerar que son dimensiones que pueden ayudar a explicar las motivaciones de uso de estos videojuegos. 
Las variaciones en el estado de ánimo permiten aproximarse al componente emocional del disfrute (Bartsch \& Oliver, 2011). Al respecto, PérezSánchez y Torres (2012) indagaron la relación entre el uso de videojuegos con diferentes intensidades de activación demandada (motora, fuerza requerida, cálculo o coordinación visomotora) sobre la activación fisiológica percibida y los estados de ánimo, y encontraron que el juego de alta intensidad favorece el aumento de los afectos positivos, no así los afectos negativos. Roger, Bowman \& Oliver (2015) encuentran que las características de la interfaz van a promover o limitar el disfrute, con el resultado de que los mandos tradicionales de las consolas generan mayor disfrute que los mandos por control de movimiento.

Junto con estas variables se considera relevante introducir el transporte emocional referido a la capacidad de las personas de situarse emocionalmente en el escenario de interacción o comunicación propios del contenido mediático al que están siendo expuestas (Burrows \& Blanton, 2016; Green \& Brock, 2000). Desde el punto de vista de los investigadores, el transporte sería el resultado de la capacidad de empatía y la toma de perspectiva, en consideración a los afectos como reguladores para la acción, de forma tal que les permiten a las personas sumergirse en un contenido mediático y definir con ello las posibilidades del intercambio comunicativo y los posibles efectos persuasivos de estos contenidos.

El altruismo, por su parte, se refiere a la ayuda voluntaria producto de las necesidades y la situación de otras personas, motivadas tanto por normas sociales como por la simpatía hacia la persona o grupo objeto de la ayuda (Eisenberg \& Fabes, 1998). Se ha encontrado tras el uso de los videojuegos que promueve comportamientos altruistas favorece las cogniciones prosociales, aunque este resultado puede estar supeditado a que en el juego la conducta altruista sea recompensada (Iten, Bopp, Steiner, Opwis \& Mekler, 2018). Esta investigación se concentra específicamente en el estudio de las variaciones en las actitudes hacia el altruismo luego de jugar dos diferentes tipos de videojuegos.

Los cambios que se puedan encontrar en estas variables producto del uso de diferentes tipos de videojuegos se han explicado como produc- 
to de la satisfacción de necesidades psicológicas básicas, a partir de lo esbozado por la teoría motivacional de la autodeterminación (Ryan \& Deci, 2000). Esta teoría parte del supuesto de que la consecución del disfrute es una motivación intrínseca que se dirige a satisfacer alguna de tres necesidades psicológicas básicas en el ser humano: la autonomía (referido a la expresión de voluntad e independencia al actuar), la filiación (la tendencia a sentirse conectado con otros) y la competencia (la necesidad de sentirse con la capacidad necesaria para actuar eficazmente) (Tamborini, Bowman, Eden, Grizzard \& Organ, 2010). Roges (2017) y Sailer, Hense, Mayr \& Mandlhan (2017) han encontrado que las características que presenta el tipo de juego en su contenido, reglas y narrativa contribuyen a la satisfacción de necesidades psicológicas específicas.

Cabe destacar que el uso de videojuegos puede variar entre hombres y mujeres. Por ejemplo, en cuanto a la frecuencia de uso, se ha encontrado que son las mujeres quienes juegan menos videojuegos y dedican menos tiempo a esa actividad (Johnson, Gardner \& Sweetser, 2016; Lucas \& Sherry, 2004; Ogletree \& Drake, 2007; Puerta-Cortes, Carbonell, Panovab \& Chamarro, 2017). La tenencia de una consola en el hogar podría tener implicaciones para el uso de videojuegos, por ejemplo, con respecto al nivel de familiarización con el equipo y de experiencia previa que tenga la persona.

En atención a todas las variables mencionadas, se consideraron las siguientes hipótesis:

H1: El juego violento presenta puntajes más altos en afectividad positiva en comparación con el juego colaborativo.

$\mathrm{H} 2$ : El juego colaborativo presenta puntajes más altos en transporte en comparación con el juego violento.

H3: El juego colaborativo presenta puntajes más altos en altruismo en comparación con el juego violento.

H4: Los puntajes en el estado de ánimo positivo para el videojuego violento se asociarán con la tenencia de una consola de videojuegos en casa. 
H5: Los puntajes en transporte emocional al jugar el videojuego colaborativo se asociarán positivamente con filiación y con la tendencia de uso eudaimónica, así como con la tenencia de consola en casa.

H6: Los puntajes en el altruismo al jugar el videojuego colaborativo se asociarán positivamente con filiación y con la tendencia de uso eudaimónica, pero negativamente con la tendencia de uso hedonista.

H7: Los puntajes en altruismo al jugar el videojuego violento se asociarán positivamente con competencia.

\section{Método}

Se llevó a cabo un diseño intrasujetos de modo que cada participante debía pasar por ambas condiciones experimentales (es decir, el tipo de videojuego). Se realizó un contrabalanceo del tipo de juego, de forma tal que se asignaron aleatoriamente los participantes que iniciaban con el videojuego colaborativo o con el videojuego violento.

\section{Participantes}

Se trabajó con 50 estudiantes universitarios, el $48 \%$ mujeres y una edad promedio de 20,6 (DT = 1,83). Los participantes debían ser usuarios frecuentes de videojuegos y haber jugado con la consola Wii. Se definió como uso frecuente al jugar al menos una vez al mes.

Cada participante firmó un consentimiento informado en el que se aclaraba el objetivo del estudio, el carácter voluntario de la participación, así como la confidencialidad en el manejo de los datos.

\section{Instrumentos}

\section{Altruismo}

Se utilizó la subescala de altruismo de la prosocial tendencies measure (PTM) de Carlo \& Randall (2002), en la versión para Costa Rica de Garbanzo y Rivera (2010). La medida consta de cuatro ítems a ser calificados en una escala Likert de 5 puntos, redactados de forma inversa, es decir, los bajos puntajes corresponden a altos niveles de altruismo. Ejemplo de los ítems 
es "Creo que si ayudo a alguien en el futuro ellos me deben ayudar", "Una de las mejores cosas de hacer trabajos caritativos es que luce bien en $\mathrm{mi} \mathrm{cu}$ rriculum". Se obtuvo un coeficiente alfa de Cronbach de 0,83.

\section{Estado de ánimo}

Los cambios en el estado de ánimo fueron medidos mediante la "Lista de afectos positivos y negativos" (Positive and Negative Affect Schedule [PANAS]) desarrollada por Watson, Clark \& Tellegen (1988), en la versión para Costa Rica de Luszczynska, Gutiérrez-Doña \& Schwarzer (2005). El instrumento está compuesto por dos subescalas con 10 ítems cada una, afecto positivo (ejemplo de ítems: orgulloso de mí, interesado) y afecto negativo (ejemplo de ítems: irritable, avergonzado). Los ítems deben calificarse en una escala de 5 puntos según el grado de presencia de cada afecto (de mucho a nada). A efectos de este estudio se recurrió a la subescala de afecto positivo. Se obtuvieron coeficientes alfa de Cronbach de 0,87.

\section{Escala de necesidades psicológicas básicas}

Esta medida fue desarrollada por Ryan, Rigby \& Przybylski (2006), a partir de los presupuestos de la teoría de la autodeterminación, compuesta por 21 ítems y 3 subescalas: autonomía ( 7 ítems), competencia ( 6 ítems) y afiliación ( 8 ítems). Se utiliza una escala de calificación de 7 puntos ( 1 no es cierto para nada, a 7 muy cierto). La escala fue modificada para este estudio. Se obtuvo un coeficiente alfa de Cronbach para competencia de 0,80, para filiación de 0,83 y para autonomía de 0,73. Ejemplos de los ítems: "He sido capaz de aprender recientemente nuevas habilidades interesantes" (competencia), "Las personas suelen ser amigables conmigo" (filiación), "Cuando juego siento que puedo ser yo mismo” (autonomía).

\section{Escala de tendencias morales de uso de videojuegos}

El instrumento fue desarrollado para esta investigación, el cual pretende medir los usos autopresentativos o prosociales de los videojuegos. Está compuesto de dos subescalas: la subescala de uso hedonista que se dirige a estudiar los usos orientados a afirmar las propias competencias al jugar y se orienta al gane. Ejemplos de ítems: "Juego porque me gusta ganar", "Me enoja cuando pierdo en el juego". La subescala prosocial se orienta a medir los usos dirigidos a colaborar y a preferir el juego colectivo o en pares. Ejemplos de ítems: 
"Me gusta jugar con otras personas", "Es divertido jugar con otras personas, aunque nos equivoquemos mientras jugamos". Los participantes indican qué tan frecuentemente hacen estas acciones siguiendo una escala Likert de 1 a 7 , de modo que es 1 "para nada frecuente" y 7 "muy frecuente".

La subescala hedonista obtuvo un coeficiente alfa de Cronbach de 0,79 , mientras que la prosocial de 0,71 .

\section{Tenencia de consola en el hogar}

Se les preguntó si poseían alguna consola en sus lugares de habitación, a lo que debían responder sí o no.

\section{Transporte emocional}

El transporte emocional se midió recurriendo a la escala de Green \& Brock (2000), adaptada por García y Pérez (en preparación), para noticias al contexto costarricense y adaptada para videojuegos en este estudio. Se trata de un instrumento de 16 ítems con una escala de respuesta, de modo que es 1 un no aplica nada y 5 aplica mucho. El instrumento mide el grado de implicación mental, emocional y la atención que se presta al contenido durante su uso. Ejemplos de ítems: "Me imaginaba a mí mismo como parte de los personajes del juego" y "Tengo toda mi atención puesta en el juego mienstras juego". Obtuvo un índice de alfa de Cronbach de 0,74.

\section{Procedimiento}

\section{Selección de los estímulos (juegos)}

Se recurrió a la consola de videojuegos Wii de Nintendo, por lo que los juegos son compatibles con dicha consola. Para indagar el uso hedonista, se escogieron juegos de tipo violentos, ya que su finalidad es competir y ganar sobre otro contrincante, además de que se busca en el contexto del videojuego salir con vida al jugar. Para el uso eudaimónico, se recurrió a un videojuego de tipo colaborativo, ya que su objetivo es avanzar en los diferentes niveles que conforma el juego con la ayuda recíproca de los jugadores. También implica considerar y respetar las habilidades de cada jugador y el tiempo que a cada uno le toma realizar las diferentes tareas que demanda el juego. 
Para seleccionar los videojuegos, se tomaron los siguientes criterios: el videojuego violento tenía que tener como objetivo, exclusivamente, la agresión física entre personajes, es decir, causar daño físico entre los personajes, como medio para mantenerse en el juego y pasar de un nivel a otro. Mientras que el juego colaborativo se debía orientar a avanzar sobre un escenario en el que fuera necesario recurrir a la ayuda de otro jugador, para resolver tareas y avanzar de un nivel o escenario a otro. Además, la resolución de tareas no debía contener agresión física.

La selección de los videojuegos, tanto el violento como el colaborativo, fue llevada a cabo por tres jueces que calificaron tres videojuegos para cada condición. Dentro de los criterios para la selección interesó evaluar, con una escala de 0 a 10, qué tanto el videojuego favorecía el juego colaborativo y qué tanto el competitivo. Si se dirigía a agredir al otro jugador o a lograr una meta en común con este. Si el disfrute se orientaba a vencer al otro jugador o a resolver una tarea en común. Además, evaluar si el juego pretendía provocar afectos positivos a través del juego violento o a través de la consecución conjunta de tareas, así como si el juego se dirigía a la satisfacción de la necesidad psicológica de filiación o, por su parte, a la afirmación de las propias necesidades.

Finalmente, se escogieron los videojuegos que obtuvieron mayor puntaje. Para el juego colaborativo, se seleccionó Lego Harry Potter que inicia contando la historia de cómo Harry llega a Hogwarts. La idea es que, mientras la historia se va contando, quienes juegan pueden tomar lecciones de magia, probar conjuros, combinar pócimas, volar con las escobas voladoras y completar tareas para ganar recompensas, y así superar las etapas de manera conjunta.

Para el juego violento, se seleccionó Mortal Kombat: Armageddon, un juego de lucha que cuenta con 60 personajes a elegir de toda la saga Mortal Kombat, centrado en debilitar y aniquilar al personaje contrincante.

\section{Procedimiento de recolección y análisis}

En el tiempo 1, los sujetos fueron asignados al azar a una de las condiciones experimentales, a saber: videojuego violento o videojuego colabora- 
tivo. En el tiempo 2, las personas realizaron la tarea correspondiente a la otra condición.

Antes de iniciar cada tiempo del experimento, los participantes debieron completar la medida de frecuencia de afecto positivo, la medida de tendencias hedonistas o eudaimónicas de uso de videojuegos y la escala de satisfacción de necesidades psicológicas básicas. Para cada condición, y dada la existencia de diferencias en cuanto al uso de videojuegos entre hombres y mujeres, las personas jugaron en parejas del mismo sexo con otra persona participante en el estudio. Inicialmente, jugaron 5 minutos de entrenamiento y, posteriormente, 15 minutos.

En los estudios precedentes que recurren a diseños experimentales como este, no es posible identificar un tiempo predefinido o estándar de juego. De ahí que se realizó un estudio piloto y se determinó un tiempo de juego similar a ambos juegos, que permitiera jugar y responder a diferentes tareas o acciones dentro de un mismo nivel o escenario. Por ello finalmente se definió 15 minutos como tiempo de juego.

Finalizada la fase de juego, se les solicitó llenar la escala de altruismo. Entre el tiempo 1 y 2 debieron pasar al menos 3 días y máximo una semana. Las personas jugaron con la misma pareja en ambos tiempos.

Para el análisis estadístico, se recurrió a pruebas ty a análisis de regresión lineal jerárquica, método paso a paso (stepwise).

\section{Resultados}

Recurriendo a las pruebas t para muestras relacionadas, se buscó determinar diferencias entre haber jugado un juego violento y un juego colaborativo sobre el estado de ánimo, el transporte emocional y la tendencia a respaldar conductas altruistas. Se encontraron diferencias en las dos primeras variables, a saber: el juego violento (condición hedonista) presenta un aumento del afecto positivo con respecto al juego colaborativo (condición eudaimónica). $t=-3,72$, yo pondría acá algo así: tal que el juego violento mostró una media de $\mathrm{p}<0,01 . \mathrm{M}_{\text {jugo violento }}=0,33 \mathrm{y}$ el juego colaborativo 
una media de $M_{\text {juego colaborativo }}=0,09$. Además, el juego colaborativo presenta mayor transporte emocional que el juego violento. $t=2,36, \mathrm{p}<0,05 . \mathrm{M}_{\text {jue- }}$ go violento $=2,47$ y $\mathrm{M}_{\text {juego colaborativo }}=2,96$.

De igual manera, resultó relevante determinar algunas variables predictoras de importancia para la comprensión de las variaciones en el transporte, el altruismo y el estado de ánimo en un tipo de juego y en otro, para lo cual se recurrió a una regresión lineal jerárquica utilizando el método paso a paso. Como variables predictoras se consideraron el sexo, la tenencia de consola de videojuegos en la casa, las tendencias de uso hedonistas o eudaimónicas ylas necesidades psicológicas básicas que pueden estar implicadas al jugar videojuegos. Para sexo y la tenencia de consola, se utilizó la codificación dummy o ficticia, que permite la introducción de variables dicotómicas en un modelo de regresión lineal (Tabachnik \& Fidell, 2007). De esta manera, en lo referente al sexo, $0=$ hombre y $1=$ mujer, y en el caso de la tenencia, 0 = posee consola y $1=$ no posee consola.

De esta manera, se encontró que predictores del transporte emocional al jugar el videojuego violento fueron no tener consola en casa $(\beta-0,32$, $\mathrm{p}<0,05 . t=2,43, \mathrm{p}<0,05, \mathrm{IC}=-1,23,-0,12)$ y ser mujer $(\beta-0,31$, $\mathrm{p}<0,05 . t=2,13, \mathrm{p}<0,05$, IC $(95 \%)=-0,84,-0,07)$. Las otras variables en el modelo de tendencias morales y necesidades quedaron excluidas $\left(\mathrm{R}^{2}\right.$ $=0,20, \mathrm{~F}(\mathrm{gl}=2,49)=6,07, \mathrm{p}<0,001)$. Por otro lado, predictores del transporte emocional en el juego colaborativo fue la necesidad de filiación ( $\beta$ $0,29, \mathrm{p}<0,05 . t=2,39, \mathrm{p}<0,05$, IC $(95 \%)=0,03,0,43)$. De modo que las personas que se orientan al uso de videojuegos como medio de integración social y mantenimiento de las relaciones de amistad tienden a presentar mayores puntajes en transporte al jugar el juego colaborativo. Las otras dimensiones quedaron excluidas $\left(\mathrm{R}^{2} 0,09, \mathrm{~F}=(\mathrm{gl}=1,49) 4,57, \mathrm{p}<0,05\right)$.

En lo que se refiere a los predictores del altruismo ante el videojuego violento, la única variable que presentó una asociación fue la necesidad de filiación $(\beta 0,33, \mathrm{p}<0,05 . t=2,34, \mathrm{p}<0,05$, IC $(95 \%)=0,05,0,68)$. Las otras dimensiones quedaron excluidas $\left(\mathrm{R}^{2} 0,16, \mathrm{~F}=(\mathrm{gl}=2,47) 5,59, \mathrm{p}<0,01\right)$. Esto quiere decir que los puntajes altos en altruismo después de jugar este 
juego violento se presentan en personas que orientan el uso de videojuegos para satisfacer la necesidad psicológico de integración social. Lo mismo sucedió para el caso del videojuego colaborativo en el que la necesidad de filiación $(\beta 0,28, \mathrm{p}<0,05 . t=-2,56, \mathrm{p}<0,05$, IC $=-0,63,-0,04)$, pero también la edad $(\beta-0,33, \mathrm{p}<0,05 . t=-2,36, \mathrm{p}<0,05$, IC $(95 \%)=-0,38$, $-0,04)$, presentaron una asociación positiva con el altruismo. Las otras dimensiones quedaron excluidas $\left(\mathrm{R}^{2} 0,21, \mathrm{~F}=(\mathrm{gl}=2,47) 6,35, \mathrm{p}<0,01\right)$.

\section{Discusión}

Se encontraron evidencias que respaldan las hipótesis 1 y 2 . Así, se pudo determinar que, después de jugar el juego violento, se presentan mayores estados de ánimo positivos en comparación con el juego colaborativo. Asimismo, se encontró mayor presencia de transporte emocional jugar el videojuego colaborativo en comparación con el juego violento. No se encontraron diferencias entre los dos tipos de videojuegos sobre los puntajes de altruismo.

Se encontraron evidencias parciales para la hipótesis 5 al encontrarse una asociación positiva entre la variable transporte y el juego violento. Además, se halló que la necesidad de filiación se asoció positivamente con la presencia de transporte emocional tras el juego colaborativo.

Se presentaron evidencias parciales para la hipótesis 6 al encontrarse una relación positiva entre los puntajes de altruismo en la necesidad de filiación. Es interesante el resultado hallado que señala que las personas con puntajes altos en la necesidad de filiación también tienen una tendencia a presentar puntajes altos en altruismo al jugar videojuegos violentos, pues estas tendencias podrían estar asociadas más bien al jugar videojuegos colaborativos. No se encontraron resultados que respalden las hipótesis 4 y 7. Además de que las tendencias de uso hedonistas o eudaimónicas no se asociaron con ninguna de las dimensiones en estudio.

En consonancia con los hallazgos encontrados por Pérez-Sánchez y Torres (2014), en relación con los videojuegos de alta demanda de activación emocional, los videojuegos violentos evocan un aumento en la activación fisiológica, lo que, junto al hecho de que se juegue en parejas, permite comprender la mejora en los afectos positivos. 
El hecho de que se encontrara mayor transporte emocional en el videojuego colaborativo puede estar relacionado precisamente con la necesidad de cooperar y coordinar las acciones como requisito para avanzar en el juego, lo que promueve un aumento en la atención y en la sensación de ser partícipe del escenario y de las acciones que demanda el videojuego. Ahora bien, esto no quiere decir que el videojuego violento estudiado no esté asociado con transporte; por el contrario, es una condición necesaria para jugarlo, aunque el videojuego colaborativo evoque comparativamente más de esta respuesta emocional. El hecho de que se presente mayor presencia de transporte en el caso de las mujeres puede deberse a que se trata de un tipo de juego que es menos practicado por este grupo (Johnson et al., 2016; Lucas \& Sherry, 2004; Ogletree \& Drake, 2007; Puerta-Cortés, et al., 2017), de ahí que se requiera un mayor uso de recursos sociocognitivos.

La satisfacción de la necesidad de filiación en el contexto de los videojuegos como predictor del altruismo viene a ser un resultado relevante para la comprensión del uso de esta tecnología, ya que sugiere que en las personas que los usan con el objetivo de la integración social y el mantenimiento de relaciones van a presentar cogniciones prosociales independiente del tipo de videojuego. Este resultado es consistente con estudios previos (Roges, 2017; Sailer, Hense, Mayr \& Mandlhan, 2017) que señalan que las particularidades de juego, su contenido, forma de juego y reglas facilitan la satisfacción de necesidades psicológicas específicas. Este resultado da cuenta de la importancia tanto del contexto social del uso como de los factores motivacionales en los efectos para el estudio de los efectos sociocognitivos, aspecto que requiere mayor investigación.

Estos resultados, además, aportan evidencias al señalamiento hecho por investigaciones previas en el sentido de que el videojuego violento por sí solo no reduce las cogniciones o comportamientos altruistas, sino que, por el contrario, deben considerarse las características de las personas usuarias de los videojuegos; en el caso de este estudio, los factores motivacionales (Fergusson \& Beresin, 2017). Es decir, parece que, si la disposición a jugar un juego en duplas está asociada a la necesidad de integración social, jugar videojuegos violentos parece no reducir las cogniciones altruistas, esto requiere, sin embargo, mayor investigación. 
De igual forma, y a manera de recomendación para futuras investigaciones, se considera que podrían realizarse estudios en la misma línea en la que los participantes puedan jugar en parejas mixtas, como forma de seguir probando la existencia de diferencias según el sexo para el uso de los videojuegos en esta población.

A partir del hecho de que no se encontraron relaciones estadísticamente significativas respecto de las dimensiones de competencia y autonomía en este estudio, se recomienda continuar el trabajo indagando el papel que están desempeñando estas necesidades psicológicas básicas en el uso de videojuegos en población adulta joven costarricense, así como cuáles pueden ser los determinantes contextuales o sociocognitivos que definen su participación como motivadores del juego.

En lo que respecta al altruismo, un condicionante del resultado encontrado puede deberse a que la medida empleada no logra ser reactiva a cambios a corto plazo, elemento que puede explicar que no se hayan encontrado diferencias luego de jugar los diferentes videojuegos.

Es importante recurrir a otro tipo de medidas o complementar con otro tipo de estudios que superen las limitaciones del autorreporte. Por ejemplo, en investigaciones futuras, resultaría de gran relevancia hacer uso de otras medidas, como la respuesta psicofisiológica, el estudio del correlato neuronal, o la investigación cualitativa de corte etnográfica o narrativa, que aporten diferentes fuentes de información, para una mejor comprensión de la actividad de las personas al jugar este tipo de videojuegos.

\section{Referencias}

Anderson, C. A., Shibuya, A., Ihori, N., Swing, E. L., Bushman, B. J., Sakamoto, A. ... Saleem, M. (2010). Violent video game effects on aggression, empathy, and prosocial behavior in Eastern and Western countries: A meta-analytic review. Psychological Bulletin, 136(2), 151-173. https://doi.org/10.1037/a0018251 
Bartsch, A. \& Oliver, M. (2011). Making sense of entertainment: On the interplay of emotion and cognition in entertainment experience. Journal of Media Psychology, 23(1), 12-17. https://doi. org/10.1027/1864-1105/a000026

Bryant, J., Roskos-Ewoldsen, D. R. \& Cantor, J. (Eds.). (2003). Communication and emotion: Essays in honor of Dolf Zillmann. New Jersey, EE. UU.: Lawrence Erlbaum.

Burrows, C. N. \& Blanton, H. (2016). Real-world persuasion from virtualworld campaigns: How transportation into virtual worlds moderates in-game influence. Communication Research, 43(4), 542-570. https://doi.org/10.1177/0093650215619215

Carlo, G. \& Randall, B. A. (2002). The development of a measure of prosocial behaviors for late adolescents. Journal of Youth AND Adolescence, 31(1), 31-44. https://doi.org/10.1023/A:1014033032440

Drummond, A., Sauer, J. D. \& Garea, S. S. (2018). The infamous relationship between violent video game use and aggression: Uncharted moderators and small effects make it a far cry from certain. En C. Ferguson (Eds.), Video game influences on aggression, cognition, and attention. (pp. 23-40). Cham, Alemania: Springer. https:// doi.org/10.1007/978-3-319-95495-0_3

Eden, A., Grizzard, M. \& Lewis, R. J. (2012). Moral psychology and media theory: Historical and emerging viewpoints. En R. Tamborini (Ed.), Media and the moral mind. (pp. 25-49). Nueva York, EE. UU.: Routledge.

Eisenberg, N., Fabes, R. A. \& Spinrad, T. L. (1998). Prosocial development. En W. Damon \& N. Eisenberg (Eds.), Handbook of child psychology: Vol. 3. Social, emotional, and personality development. (5. ${ }^{\text {a }}$ ed., pp. 701-778). Nueva York, EE. UU.: Wiley. https://doi. org/10.1002/9780470147658.chpsy0311 
Ferguson, C. J. \& Beresin, E. (2017). Social science's curious war with pop culture and how it was lost: The media violence debate and the risks it holds for social science. Preventive Medicine, 99, 69-76. https: // doi.org/10.1016/j.ypmed.2017.02.009

Garbanzo Rodríguez, G. y Rivera Villareal, C. (2010). El uso de un juego de simulación digital comercial como herramienta pedagógica en el aprendizaje de la informática en un grupo de adultos del cantón de Curridabat (Tesis de grado, Universidad de Costa Rica, San José de Costa Rica). http://sipub.inie.ucr.ac.cr/SIPUB/detallesResultado.do?idProduccion $=1127$

Green, M. C. \& Brock, T. C. (2000). The role of transportation in the persuasiveness of public narratives. Journal of Personality and Social Psychology, 79(5), 701-721. https://doi.org/10.1037/00223514.79.5.701

Greitemeyer, T. \& Osswald, S. (2009). Prosocial video games reduce aggressive cognitions. Journal of Experimental Social Psychology, 45(4), 896-900. https://doi.org/10.1016/j.jesp.2009.04.005

Greitemeyer, T., Agthe, M., Turner, R. \& Gschwendtner, C. (2012). Acting prosocially reduces retaliation: Effects of prosocial video games on aggressive behavior. European Journal of Social Psychology, 42(2), 235-242. https://doi.org/10.1002/ejsp.1837

Greitemeyer, T., Osswald, S. \& Brauer, M. (2010). Playing prosocial video games increases empathy and decreases schadenfreude. Emotion, 10(6), 796-802. https://doi.org/10.1037/a0020194

Hollingdale, J. \& Greitemeyer, T. (2014). The effect of online violent video games on levels of aggression. PLoS One, 9(11), e111790.https:// doi.org/10.1371/journal.pone.0111790

Iten, G. H., Bopp, J. A., Steiner, C., Opwis, K. \& Mekler, E. D. (2018). Does a prosocial decision in video games lead to increased prosocial 
real-life behavior? The impact of reward and reasoning. Computers in Human Behavior, 89, 163-172. https://doi.org/10.1016/j. chb.2018.07.031

Jerabeck, J. M. \& Ferguson, C. J. (2013). The influence of solitary and cooperative violent video game play on aggressive and prosocial behavior. Computers in Human Behavior, 29(6), 2573-2578. https:// doi.org/10.1016/j.chb.2013.06.034

Johnson, D., Gardner, J. \& Sweetser, P. (2016). Motivations for videogame play: Predictors of time spent playing. Computers in Human Behavior, 63, 805-812. https://doi.org/10.1016/j.chb.2016.06.028

Lewis, R. J., Tamborini, R. \& Weber, R. (2014). Testing a dual-process model of media enjoyment and appreciation. Journal of Communication, 64(3), 397-416. https://doi.org/10.1111/jcom.12101

Lucas, K. \& Sherry, J. L. (2004). Sex differences in video game play: A communication-based explanation. Communication Research, 31(5), 499-523. https://doi.org/10.1177/0093650204267930

Luszczynska, A., Gutiérrez-Doña, B. \& Schwarzer, R. (2005). General selfefficacy in various domains of human functioning: Evidence from five countries. International Journal of Psychology, 40(2), 80-89. https://doi.org/10.1080/00207590444000041

Ogletree, S. M. \& Drake, R. (2007). College students' video game participation and perceptions: Gender differences and implications. Sex Roles, 56(7-8), 537-542. https://doi.org/10.1007/s11199007-9193-5

Oliver, M. B. \& Bartsch, A. (2010). Appreciation as audience response: Exploring entertainment gratifications beyond hedonism. Human Communication Research, 36(1), 53-81.https://doi.org/10.1111/ j.1468-2958.2009.01368.x 
Pérez-Sánchez, R. y Torres, D. (2014). Intensidad de demanda de los videojuegos y su efecto sobre el estado de ánimo y la activación percibida. Universitas Psychologica, 13(4), 1489-1502. https://doi. org/10.11144/Javeriana.UPSY13-4.idve

Puerta-Cortés, D. X., Panova, T., Carbonell, X. \& Chamarro, A. (2017). How passion and impulsivity influence a player's choice of videogame, intensity of playing and time spent playing. Computers in Human Behavior, 66, 122-128.https://doi.org/10.1016/j.chb.2016.09.029

Rogers, R. (2017). The motivational pull of video game feedback, rules, and social interaction: Another self-determination theory approach. Computers in Human Behavior, 73, 446-450. https://doi. org/10.1016/j.chb.2017.03.048

Rogers, R., Bowman, N. D. \& Oliver, M. B. (2015). It's not the model that doesn't fit, it's the controller! The role of cognitive skills in understanding the links between natural mapping, performance, and enjoyment of console video games. Computers in Human Behavior, 49, 588-596. https://doi.org/10.1016/j.chb.2015.03.027

Ryan, R. M. \& Deci, E. L. (2000). Self-determination theory and the facilitation of intrinsic motivation, social development, and well-being. American Psychologist, 55(1), 68-78. http://doi. org/10.1037/0003-066X.55.1.68

Ryan, R. M., Rigby, C. S. \& Przybylski, A. (2006). The motivational pull of video games: A self-determination theory approach. Motivation and Emotion, 30(4), 344-360. https://doi.org/10.1007/s11031006-9051-8

Sailer, M., Hense, J. U., Mayr, S. K. \& Mandl, H. (2017). How gamification motivates: An experimental study of the effects of specific game design elements on psychological need satisfaction. Computers in Human Behavior, 69, 371-380. https://doi.org/10.1016/j. chb.2016.12.033 
Saleem, M., Anderson, C. A. \& Gentile, D. A. (2012). Effects of prosocial, neutral, and violent video games on college students' affect. Aggressive Behavior, 38(4), 263-271.https://doi.org/10.1002/ab.21427

Tabachnick, B. G. \& Fidell, L. S. (2007). Using multivariate statistics. (5.a ed.). Boston, EE. UU.: Pearson.

Tamborini, R., Bowman, N. D., Eden, A., Grizzard, M. \& Organ, A. (2010). Defining media enjoyment as the satisfaction of intrinsic needs. Journal of Communication, 60(4), 758-777. https://doi. org/10.1111/j.1460-2466.2010.01513.x

Watson, D., Clark, L. A. \& Tellegen, A. (1988). Development and validation of brief measures of positive and negative affect: The PANAS scales. Journal of Personality and Social Psychology, 54(6), 1063-1070. https://doi.org/10.1037/0022-3514.54.6.1063

Zillmann, D. (1988). Mood management through communication choices. American Behavioral Scientist, 31(3), 327-340. https://doi. org/10.1177/000276488031003005 